\title{
Bioinformatic analysis identifies potential biomarkers and therapeutic targets of septic- shock-associated acute kidney injury
}

\author{
Yun Tang ${ }^{\dagger}$, Xiaobo Yang ${ }^{\dagger}$, Huaqing Shu, Yuan Yu, Shangwen Pan, Jiqian Xu and You Shang ${ }^{*}$
}

\begin{abstract}
Background: Sepsis and septic shock are life-threatening diseases with high mortality rate in intensive care unit (ICU). Acute kidney injury (AKI) is a common complication of sepsis, and its occurrence is a poor prognostic sign to septic patients. We analyzed co-differentially expressed genes (co-DEGs) to explore relationships between septic shock and AKI and reveal potential biomarkers and therapeutic targets of septic-shock-associated AKI (SSAKI).

Methods: Two gene expression datasets (GSE30718 and GSE57065) were downloaded from the Gene Expression Omnibus (GEO). The GSE57065 dataset included 28 septic shock patients and 25 healthy volunteers and blood samples were collected within 0.5, 24 and $48 \mathrm{~h}$ after shock. Specimens of GSE30718 were collected from 26 patients with AKI and 11 control patents. AKI-DEGs and septic-shock-DEGs were identified using the two datasets. Subsequently, Gene Ontology (GO) functional analysis, Kyoto Encyclopedia of Genes and Genomes (KEGG) pathway enrichment analysis, and protein-protein interaction (PPI) network analysis were performed to elucidate molecular mechanisms of DEGs. We also evaluated co-DEGs and corresponding predicted miRNAs involved in septic shock and AKl.

Results: We identified 62 DEGs in AKI specimens and 888, 870, and 717 DEGs in septic shock blood samples within $0.5,24$ and $48 \mathrm{~h}$, respectively. The hub genes of EGF and OLFM4 may be involved in AKI and QPCT, CKAP4, PRKCQ, PLAC8, PRC1, BCL9L, ATP11B, KLHL2, LDLRAP1, NDUFAF1, IFIT2, CSF1R, HGF, NRN1, GZMB, and STAT4 may be associated with septic shock. Besides, Co-DEGs of VMP1, SLPI, PTX3, TIMP1, OLFM4, LCN2, and S100A9 coupled with corresponding predicted miRNAs, especially miR-29b-3p, miR-152-3p, and miR-223-3p may be regarded as promising targets for the diagnosis and treatment of SSAKI in the future.
\end{abstract}

Conclusions: Septic shock and AKI are related and VMP1, SLPI, PTX3, TIMP1, OLFM4, LCN2, and S100A9 genes are significantly associated with novel biomarkers involved in the occurrence and development of SSAKI.

Keywords: Sepsis, Septic shock, Acute kidney injury, Bioinformatic analysis, Differentially expressed genes

\footnotetext{
*Correspondence: you_shanghust@163.com

†Yun Tang and Xiaobo Yang contributed equally to this work.

Department of Critical Care Medicine, Union Hospital, Tongji Medical College, Huazhong University of Science and Technology, No.1277,

Jiefang Avenue, Wuhan 430022, China
}

(c) The Author(s) 2021. This article is licensed under a Creative Commons Attribution 4.0 International License, which permits use, sharing, adaptation, distribution and reproduction in any medium or format, as long as you give appropriate credit to the original author(s) and the source, provide a link to the Creative Commons licence, and indicate if changes were made. The images or other third party material in this article are included in the article's Creative Commons licence, unless indicated otherwise in a credit line to the material. If material is not included in the article's Creative Commons licence and your intended use is not permitted by statutory regulation or exceeds the permitted use, you will need to obtain permission directly from the copyright holder. To view a copy of this licence, visit http://creativeco mmons.org/licenses/by/4.0/. The Creative Commons Public Domain Dedication waiver (http://creativecommons.org/publicdomain/ zero/1.0/) applies to the data made available in this article, unless otherwise stated in a credit line to the data. 


\section{Background}

Sepsis is a life-threatening disease which is defined as severe organ dysfunction results from a dysregulated innate immune response following infection. Septic shock is a subset of sepsis and is associated with severe cellular, metabolic, and circulatory abnormalities [1]. Currently, sepsis is one of the major causes of death in intensive care unit (ICU), with a mortality rate ranging from 20 to $50 \%$ of all cases [2].

Acute kidney injury (AKI) is a common complication of sepsis with an incidence between 47 to $61 \%$ [3, 4]. The morbidity of AKI in the context of septic patients is a poor prognostic sign and is correlated with higher mortality, increased length of ICU stay and hence considerable healthcare resources consumption [5]. Septic AKI carries a high mortality rate of up to 70\% [6-9]. Due to its high morbidity and mortality in critically ill patients, it is of great importance to identify those septic patients at highest risk of developing AKI.

Diagnosis for septic AKI has been and is still dependent on serum creatinine and urine output [10]. However, the two functional markers have serious limitations that may lead to late or even missed diagnosis. Serum creatinine concentrations might not change until $48-72 \mathrm{~h}$ after the initial insult to the kidney, and tend to rise when about $50 \%$ of kidney function has already been lost [11]. Moreover, renal injury can exist with no change or only slightly increase in creatinine due to renal reserve or tubular secretion of creatinine [12]. In addition, an oliguria or an increase in creatinine may be also caused by renal hypoperfusion due to prerenal factors although kidney function is not impaired [13]. Consequently, the effectiveness of therapy for septic AKI is largely limited by the diagnosis that based upon changes in serum creatinine and urine output. In order to identify and develop robust diagnostic biomarkers and therapeutic strategies for septic AKI, a better understanding of the mechanisms leading to renal damage as well as recovery in septic patients is essential.

In this study, two datasets were downloaded and analyzed from the Gene Expression Omnibus (GEO) to identified genes that are co-differentially expressed (coDEGs) in septic shock and AKI. Then, we elucidated molecular mechanisms of septic-shock-related DEGs and AKI-related DEGs through functional and pathway analyses and protein-protein interaction (PPI) network analysis. Finally, we predicted microRNAs (miRNAs) that may be involved in the process of septic shock patients prone to AKI.

\section{Materials and methods Microarray data}

Two gene expression datasets (GSE30718 [14] and GSE57065 [15]) were downloaded from the GEO (http:// www.ncbi.nlm.nih.gov/geo/) [16] and the platform used for both expression profiling arrays was the GPL570 [HG-U133_Plus_2] Affymetrix Human Genome U133 Plus 2.0 Array (Affymetrix, Santa Clara, CA, USA). The two datasets have included all necessary information and no samples had to be taken on site.

The GSE57065 dataset included 28 septic shock patients and 25 healthy volunteers and blood samples were collected within $0.5,24$ and $48 \mathrm{~h}$ after shock. Patients aged $<18$, having one or more severe comorbidities, or receiving immunosuppressive therapy were excluded. The diagnosis of septic shock used the ACCP/ SCCM criteria. The onset of the septic shock was defined as the beginning of vasopressor therapy. Specimens of GSE30718 were collected from 26 patients with AKI and 11 control patents. The AKI cohort consisted of 28 biopsies from 26 kidney transplants with acute injury, with a mean estimated glomerular filtration rate (eGFR) at biopsy of $26 \mathrm{ml} / \mathrm{min}$. Controls consisted of 11 pristine protocol biopsies from stable kidney transplants with no histologic abnormalities, with a mean eGFR at biopsy of $51.2 \mathrm{ml} / \mathrm{min}$. All RNA information of the selected samples was downloaded for further analysis. Ethical approval was not necessary because our study is bioinformatic analysis.

\section{Identification of DEGs}

The original expression matrix was processed by $\mathrm{R}$ software. The "limma" package [17] was utilized to screen out DEGs. Adjusted $P$-value $<0.05$ and $\mid$ Log fold-change $\mid>1$ were used for filtering septic-shock-DEGs and AKI-DEGs. DEGs from the two datasets were screened for subsequent Gene Ontology (GO), Kyoto Encyclopedia of Genes and Genomes (KEGG) pathway enrichment analyses, and PPI network analysis. In addition, co-DEGs for AKI and septic shock were calculated and made on Venn diagram.

\section{PPI network construction and hub gene identification} PPI networks of AKI- and septic-shock-DEGs were constructed using the Search Tool for the Retrieval of Interacting Genes (STRING; http://string-db.org/). Interaction with a combined score $>0.4$ of AKI-DEGs and $>0.9$ of septic-shock-DEGs were set as the cut-off points. Subsequently, Cytoscape software was used to construct and visualize molecular interaction networks [18]. The hub genes in the PPI networks were selected using the plug-in Molecular Complex Detection (MCODE) of Cytoscape [19].

\section{GO and KEGG pathway enrichment analyses of DEGs}

$\mathrm{GO}$ function analysis (cellular component [CC], biological process $[\mathrm{BP}]$, and molecular function $[\mathrm{MF}]$ ) is a powerful bioinformatics tool to classify gene expression and 
its properties [20]. KEGG pathway analysis was used to find out which cell pathways might be involved in the changes in DEGs [21]. GO and KEGG pathway enrichment analyses of AKI- and septic-shock-DEGs were performed using the R package "clusterProfiler" [22]. A $P<0.05$ was considered statistically significant.

\section{Associations between co-DEGs and kidney or infectious diseases}

The comparative toxicogenomics database (CTD; http:// ctdbase.org/) is a public resource that describes interactions of chemical-gene/protein, chemical-disease, and gene/protein-disease, providing information about interactions between environmental chemicals and gene products and their relationships to diseases [23]. Thus, we used these data to analyze and identify the associations between co-DEGs and kidney or infectious diseases.

\section{Functional enrichment in co-DEGs}

The AmiGO database (v2.0; http://amigo.geneontolo gy.org/amigo/) is a web-based application for querying, browsing, visualizing, and downloading the Gene Ontology and annotations [24]. We used the AmiGO database to comfirm GO term enrichment for identified co-DEGs involved in AKI and septic shock.

\section{GO and KEGG pathway enrichment among predicted miRNAs and co-DEGs}

We used the STARBASE Database ENCORI (http://starb ase.sysu.edu.cn/) to predict potential microRNAs that may regulate co-DEGs [25]. Six prediction programs (PITA, miRmap, miRanda, microT, PicTar, and TargetScan) were utilized to determine candidate miRNAs of each co-DEG. Subsequently, we applied computational tool Diana-miRPath (v3.0; http://www.microrna.gr/ miRPathv3) to assess interactions among miRNAs previously identified and co-DEGs [26].

\section{Results \\ Identification of DEGs}

In total, 62 DEGs were extracted from the AKI samples, among which 22 genes were up-regulated and 40 genes were down-regulated (Additional file 1). In addition, we identified 888, 870, and 717 DEGs within $0.5,24$ and $48 \mathrm{~h}$ after septic shock, respectively (Additional file 2, Additional file 3 and Additional file 4). Here, 598 co-expressed DEGs in the three time points mentioned above were confirmed as septic-shock-DEGs. The Venn diagram were presented in Fig. 1c.

PPI network construction and hub gene identification We identified 27 nodes and 266 nodes from PPI network of AKI- and septic-shock-DEGs, respectively (Fig. 1a, b). Two hub nodes, involved in epidermal growth factor $(E G F)$ and olfactomedin 4 (OLFM4), are considering as hub genes related to AKI. A total of 16 genes, including glutaminyl-peptide cyclotransferase (QPCT), cytoskeleton-associated protein 4 (CKAP4), protein kinase $\mathrm{C}$, theta $(P R K C Q)$, placenta-specific 8 (PLAC8), protein regulator of cytokinesis 1 (PRC1), B-cell CLL/ lymphoma 9-like (BCL9L), ATPase, class VI, type $11 \mathrm{~B}$ (ATP11B), kelch-like family member 2 (KLHL2), low density lipoprotein receptor adaptor protein 1 ( $L D L$ RAP1), NADH dehydrogenase complex I, assembly factor 1 (NDUFAF1), interferon-induced protein with tetratricopeptide repeats 2 (IFIT2), colony stimulating factor 1 receptor $(C S F 1 R)$, hepatocyte growth factor $(H G F)$, neuritin $1(N R N 1)$, granzyme $\mathrm{B}(G Z M B)$, and signal transducer and activator of transcription 4 (STAT4), are identified as hub genes associated with septic shock.

\section{GO and KEGG pathway enrichment analyses of DEGs}

With respect to AKI-DEGs, the GO terms related BP were mainly enriched in neutrophil degranulation/ activation, neutrophil mediated immunity, and antimicrobial humoral response. CC were primarily associated with vesicle lumen, secretory granule lumen, and extracellular matrix. MF were mainly involved in sulfur compound binding, heparin binding, and peptidase inhibitor activity. As for septic-shock-DEGs, the BP terms of neutrophil activation/degranulation, neutrophil mediated immunity, and $\mathrm{T}$ cell activation were significantly enriched. There is significant correlation in vesicle lumen, secretory granule lumen, and specific granule in relation to CC. Similarly, MF were mainly enriched in immune receptor activity, peptidase regulator activity, and cytokine binding (Fig. 2).

KEGG pathway enrichment analysis of AKI-DEGs were mainly enriched in IL -17 signaling pathway, retinol metabolism, chagas disease, and parathyroid hormone synthesis. KEGG terms included hematopoietic cell lineage, Th1 and Th2 cell differentiation, Th17 cell differentiation, and human T-cell leukemia virus 1 infection were enriched in septic-shock-DEGs (Fig. 2).

\section{Functional enrichment in co-DEGs}

Seven co-expressed DEGs, including vacuole membrane protein 1 (VMP1), secretory leukocyte peptidase inhibitor $(S L P I)$, pentraxin 3 (PTX3), TIMP metallopeptidase inhibitor 1 (TIMP1), olfactomedin 4 (OLFM4), lipocalin 2 (LCN2), S100 calcium binding protein A9 (S100A9), were observed. The CTD database revealed that co-DEGs targeted various kidney and infectious diseases (Table 1). The AmiGO database was used to recognize GO consortium of co-DEGs and these data appear in Table 2. 

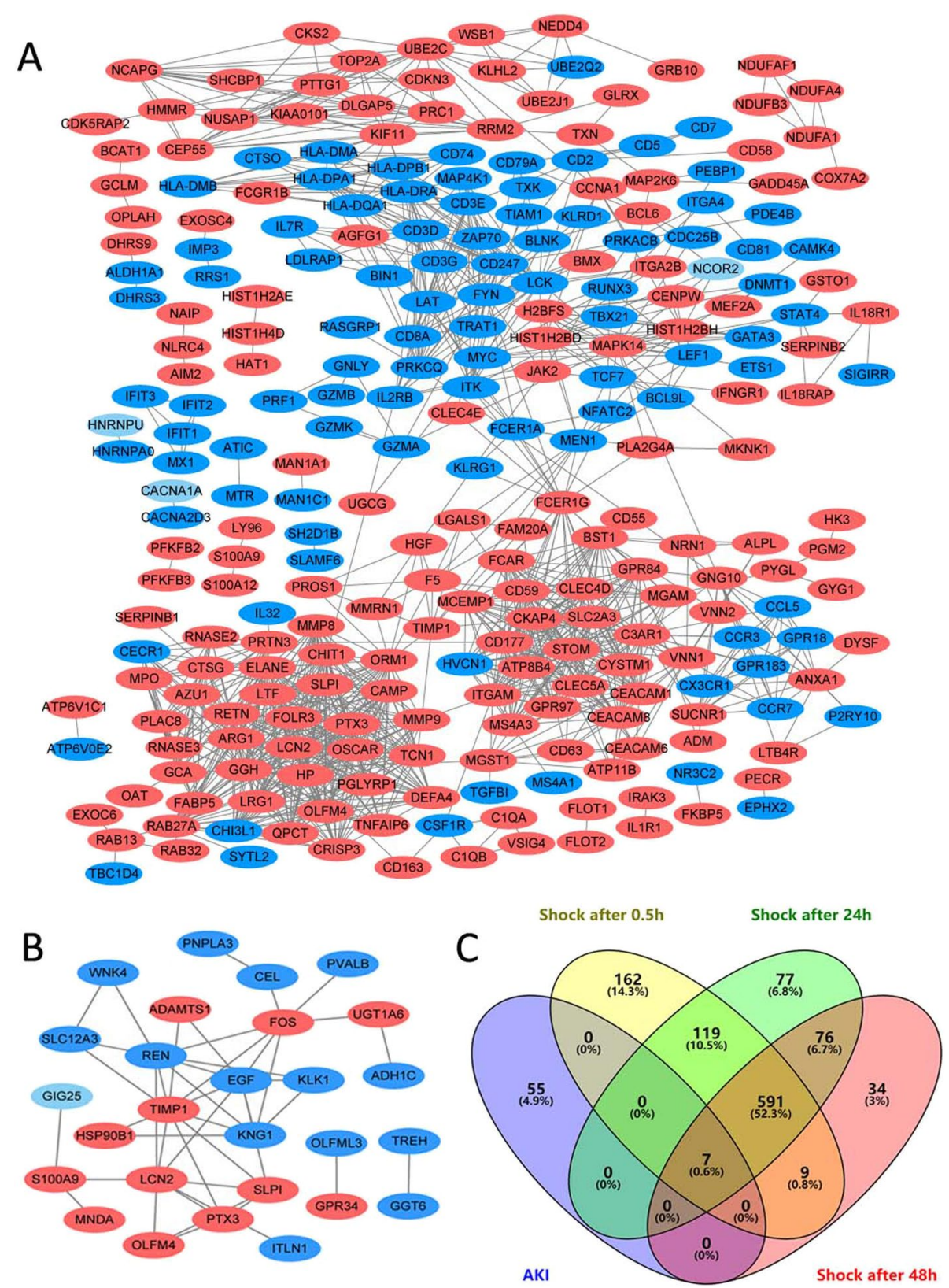

Fig. 1 PPI network and Venn diagrams. a, b The PPI networks of septic-shock-DEGs and AKI-DEGs constructed using Cytoscape. Red represents upregulated genes, and blue represents downregulated genes. c Venn diagram of DEGs related to AKI and within 0.5, 24, and 48 hafter septic shock, respectively. Seven co-expressed genes, including VMP1, SLPI, PTX3, TIMP1, OLFM4, LCN2, and S100A9, are identified

\section{GO and KEGG pathway enrichment among predicted miRNAs and co-DEGs}

The miRNAs targeting each co-DEG were selected if they were predicted by two or more prediction programs. The analyses of GO and KEGG pathway enrichment between predicted miRNAs and co-DEGs were shown in Table 3. These analyses made us clearness the molecular mechanisms how predicted miRNAs influence septic-shockassociated AKI (SSAKI).

\section{Discussion}

The mortality of AKI induced by sepsis and septic shock has remained high in recent years. Early diagnosis, appropriate classification and timely treatments in the initial periods of septic AKI play a crucial role in reducing mortality. Bioinformatic analyses enable us to understand the molecular mechanisms of disease occurrence and development, providing a novel and effective way to identify potential diagnostic biomarkers 

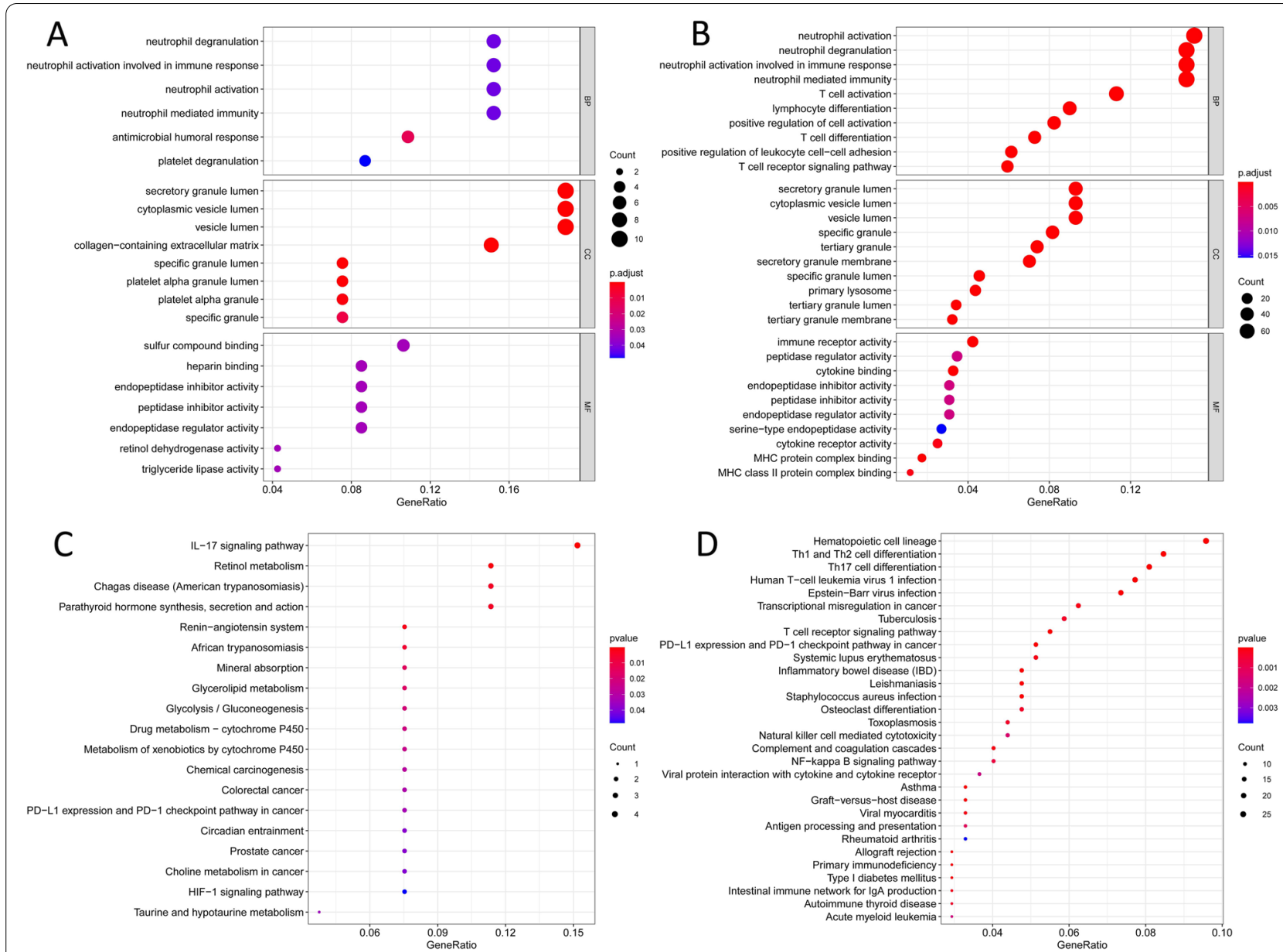

Fig. 2 GO terms and KEGG pathway enrichment. $\mathbf{a}$, b GO categories of AKI- and septic-shock-DEGs, respectively. c, d KEGG pathway enrichment of AKI- and septic-shock-DEGs, respectively

and therapeutic targets in preventing and treating septic AKI. In the present study, seven co-DEGs of septic shock and AKI, including VMP1, SLPI, PTX3, TIMP1, OLFM4, LCN2 and S100A9, were identified.

TIMP1 belongs to the TIMP gene family and the proteins encoded by this gene family are known to inhibit the matrix metalloproteinases (MMPs) activity and regulate the balance of matrix remodeling during degradation of the extracellular matrix [27]. Bojic et al. compared 53 patients with sepsis divided into sepsis-associated acute kidney injury (SAAKI) group and non-SAAKI group to 50 controls without sepsis [28]. They found that the patients with SAAKI had higher serum TIMP1 expression compared to septic patients without SAAKI and the control group. Their findings suggested TIMP1 could serve as potential diagnostic biomarker of sepsis with AKI. OLFM4 gene encodes a highly glycosylated protein which contains the olfactomedin domain [29]. The encoded protein has been proved to regulate various cellular functions such as cell growth, apoptosis, differentiation and proliferation [30]. Stark et al. reported that wild type mice had a significant increase in serum creatinine and renal cell apoptosis at $24 \mathrm{~h}$ after cecal slurry injection while it was not observed in OLFM4 null mice. This revealed that OLFM4 expression may be involved with kidney injury in sepsis [31]. A retrospective cohort study demonstrated that upregulation of OLFM4 in peripheral blood was associated with SSAKI via transcriptome analysis [32]. These findings indicated that the OLFM4 gene may be a potential biomarker and prognostic indicator for SSAKI. $L C N 2$, also known as neutrophil gelatinase-associated lipocalin $(N G A L)$, is a member of the lipocalin superfamily. The protein encoded by $L C N 2$ is involved in the transport of small hydrophobic molecules [33]. Wang et al. reported that the expression of $N G A L$ was much higher in AKI than in non-AKI septic patients and the AUC of NGAL expression for predicting AKI in septic patients was higher than that of procalcitonin (PCT) [34]. The present study proved that NGAL may be an emerging diagnostic biomarker for 
Table 1 Relationship to infectious and kidney diseases related to co-DEGs based on the CTD database

\begin{tabular}{|c|c|c|c|c|}
\hline Genes & Disease name & Disease ID & Inference score & Reference count \\
\hline \multirow[t]{6}{*}{ VMP1 } & Proteus Infections & MESH:D011512 & 7.77 & 2 \\
\hline & Acinetobacter Infections & MESH:D000151 & 4 & 1 \\
\hline & Bacterial Infections & MESH:D001424 & 3.79 & 1 \\
\hline & Kidney Diseases & MESH:D007674 & 74.98 & 274 \\
\hline & Acute Kidney Injury & MESH:D058186 & 57.3 & 164 \\
\hline & Kidney Tubular Necrosis, Acute & MESH:D007683 & 37.19 & 38 \\
\hline \multirow[t]{6}{*}{ SLPI } & Bacterial Infections & MESH:D001424 & 14.92 & 8 \\
\hline & Pneumonia, Pneumocystis & MESH:D011020 & 12.12 & 4 \\
\hline & Staphylococcal Infections & MESH:D013203 & 11.08 & 31 \\
\hline & Kidney Diseases & MESH:D007674 & 140.73 & 445 \\
\hline & Acute Kidney Injury & MESH:D058186 & 81.18 & 300 \\
\hline & Proteinuria & MESH:D011507 & 72.69 & 160 \\
\hline \multirow[t]{6}{*}{ PTX3 } & Bacterial Infections & MESH:D001424 & 10.56 & 4 \\
\hline & Helicobacter Infections & MESH:D016481 & 9.69 & 3 \\
\hline & Pseudomonas Infections & MESH:D011552 & 6.47 & 2 \\
\hline & Kidney Diseases & MESH:D007674 & 107.5 & 348 \\
\hline & Acute Kidney Injury & MESH:D058186 & 88.8 & 205 \\
\hline & Proteinuria & MESH:D011507 & 83.53 & 150 \\
\hline \multirow[t]{7}{*}{ TIMP1 } & Bacterial Infections & MESH:D001424 & 38.56 & 11 \\
\hline & IMMUNE SUPPRESSION & OMIM:146850 & 25.12 & 4 \\
\hline & Staphylococcal Infections & MESH:D013203 & 24.54 & 114 \\
\hline & Glomerulonephritis, IGA & MESH:D005922 & 11.38 & 6 \\
\hline & Kidney Diseases & MESH:D007674 & 417.32 & 804 \\
\hline & Acute Kidney Injury & MESH:D058186 & 305.13 & 549 \\
\hline & Proteinuria & MESH:D011507 & 245.56 & 439 \\
\hline \multirow[t]{6}{*}{ OLFM4 } & IMMUNE SUPPRESSION & OMIM:146850 & 4.94 & 1 \\
\hline & Hidradenitis Suppurativa & MESH:D017497 & 3.98 & 1 \\
\hline & Meningitis, Pneumococcal & MESH:D008586 & 3.65 & 1 \\
\hline & Kidney Diseases & MESH:D007674 & 52.19 & 67 \\
\hline & Acute Kidney Injury & MESH:D058186 & 33.22 & 21 \\
\hline & Proteinuria & MESH:D011507 & 31.39 & 24 \\
\hline \multirow[t]{7}{*}{ LCN2 } & Meningitis, Cryptococcal & MESH:D016919 & 32.23 & 13 \\
\hline & Mycoses & MESH:D009181 & 30.46 & 30 \\
\hline & Candidiasis, Vulvovaginal & MESH:D002181 & 22.19 & 5 \\
\hline & Kidney Diseases ${ }^{\mathrm{a}}$ & MESH:D007674 & 328.81 & 776 \\
\hline & Acute Kidney Injury ${ }^{\mathrm{a}}$ & MESH:D058186 & 227.05 & 523 \\
\hline & Proteinuria & MESH:D011507 & 177.1 & 385 \\
\hline & Renal Insufficiency & MESH:D051437 & 140.84 & 170 \\
\hline \multirow[t]{6}{*}{ S100A9 } & Bacterial Infections & MESH:D001424 & 22.04 & 8 \\
\hline & Staphylococcal Infections & MESH:D013203 & 18.13 & 15 \\
\hline & IMMUNE SUPPRESSION & OMIM:146850 & 17.92 & 4 \\
\hline & Kidney Diseases & MESH:D007674 & 274.34 & 537 \\
\hline & Acute Kidney Injury & MESH:D058186 & 214.94 & 463 \\
\hline & Renal Insufficiency & MESH:D051437 & 146.23 & 153 \\
\hline
\end{tabular}

a Direct evidence of marker or mechanism in this disease

AKI in septic patients. The protein encoded by S100A9 is a member of the $\mathrm{S} 100$ family of calcium-binding proteins that promotes and exacerbates the inflammatory response [35]. Study have reported that patients with higher serum S100A9 expression tended to suffer from more severe sepsis-related organ dysfunction [36]. Leeds et al. observed that expression of $\$ 100 A 9$ was drastically increased in iMCD3 (inner medullary collecting duct 
Table 2 The GO terms enrichment for the co-DEGs of septic shock and AKI

\begin{tabular}{|c|c|c|c|}
\hline Gene/product & GO class (direct) & Evidence & Reference \\
\hline \multirow[t]{3}{*}{ VMP1 } & protein binding & $\mid \mathrm{PI}$ & PMID:17724469 \\
\hline & endoplasmic reticulum & IDA & GO_REF:0000052 \\
\hline & autophagosome assembly & IMP & PMID:30093494 \\
\hline \multirow[t]{8}{*}{ SLPI } & protein binding & $\mid \mathrm{PI}$ & PMID:12526812 \\
\hline & antibacterial humoral response & ISS & GO_REF:0000024 \\
\hline & extracellular space & IDA & PMID:18714013 \\
\hline & collagen-containing extracellular matrix & $\mathrm{HDA}$ & PMID:28675934 \\
\hline & innate immune response & ISS & GO_REF:0000024 \\
\hline & modulation of process of other organism & IDA & PMID:2467900 \\
\hline & negative regulation of endopeptidase activity & IEA & GO_REF:0000108 \\
\hline & serine-type endopeptidase inhibitor activity & IDA & PMID:3462719 \\
\hline \multirow[t]{5}{*}{ PTX3 } & protein binding & IPI & PMID:19050261 \\
\hline & complement component C1q complex binding & IDA & PMID:23544079 \\
\hline & extracellular region & TAS & Reactome:R-HSA-6798745 \\
\hline & extracellular space & IDA & PMID:23544079 \\
\hline & innate immune response & IDA & PMID:23544079 \\
\hline \multirow[t]{8}{*}{ TIMP1 } & protein binding & IPI & PMID:16917503 \\
\hline & extracellular region & TAS & Reactome:R-HSA-1602454 \\
\hline & metalloendopeptidase inhibitor activity & IDA & PMID:12714508 \\
\hline & basement membrane & IEA & GO_REF:0000107 \\
\hline & cytokine-mediated signaling pathway & TAS & Reactome:R-HSA-6783783 \\
\hline & extracellular space & IDA & PMID:3903517 \\
\hline & negative regulation of endopeptidase activity & IDA & PMID:3903517 \\
\hline & negative regulation of membrane protein ectodomain proteolysis & IDA & PMID:12714508 \\
\hline \multirow[t]{5}{*}{ OLFM4 } & protein binding & IPI & PMID:20534456 \\
\hline & extracellular exosome & HDA & PMID:19056867 \\
\hline & extracellular space & HDA & PMID:16502470 \\
\hline & extracellular region & TAS & Reactome:R-HSA-6798745 \\
\hline & plasma membrane & IDA & GO_REF:0000052 \\
\hline \multirow[t]{5}{*}{ LCN2 } & protein binding & $\mid \mathrm{PI}$ & PMID:32296183 \\
\hline & extracellular region & ISS & GO_REF:0000024 \\
\hline & extracellular space & HDA & PMID:16502470 \\
\hline & extracellular exosome & HDA & PMID:19056867 \\
\hline & iron ion binding & ISS & GO_REF:0000024 \\
\hline \multirow[t]{13}{*}{ S100A9 } & protein binding & $\mid \mathrm{PI}$ & PMID:17620599 \\
\hline & extracellular region & TAS & PMID:22489132 \\
\hline & collagen-containing extracellular matrix & $\mathrm{HDA}$ & PMID:23979707 \\
\hline & extracellular space & HDA & PMID:16502470 \\
\hline & cytosol & IDA & GO_REF:0000052 \\
\hline & extracellular exosome & HDA & PMID:19056867 \\
\hline & antimicrobial humoral immune response mediated by antimicrobial peptide & IDA & PMID:12874352 \\
\hline & calcium ion binding & TAS & PMID:22489132 \\
\hline & cytoplasm & IDA & PMID:12874352 \\
\hline & leukocyte migration involved in inflammatory response & IDA & PMID:12626582 \\
\hline & neutrophil aggregation & IDA & PMID:12626582 \\
\hline & neutrophil chemotaxis & IDA & PMID:12626582 \\
\hline & nucleus & $\mathrm{HDA}$ & PMID:21630459 \\
\hline
\end{tabular}

IPI physical interaction evidence used in manual assertion, IDA direct assay evidence used in manual assertion, ISS sequence similarity evidence used in manual assertion, IEA evidence used in automatic assertion, TAS traceable author statement used in manual assertion, IMP mutant phenotype evidence used in manual assertion, $H A D$ high throughput direct assay evidence used in manual assertion 
Table $3 \mathrm{GO}$ and KEGG pathways enrichment among predicted miRNAs and co-DEGs

\begin{tabular}{|c|c|c|c|c|}
\hline Genes & Predicted miRNAs & Category & & $\boldsymbol{P}$ value \\
\hline \multirow[t]{3}{*}{ SLPI } & hsa-miR-370-3p & KEGG pathway & NA & \\
\hline & hsa-miR-3679-5p & GO terms & NA & \\
\hline & hsa-miR-520a-5p & & & \\
\hline \multirow[t]{11}{*}{ TIMP1 } & hsa-miR-22-3p & KEGG pathway & HIF-1 signaling pathway & $9 e-05$ \\
\hline & hsa-miR-4739 & GO terms & regulation of integrin-mediated signaling pathway & 0.002 \\
\hline & hsa-miR-1321 & & negative regulation of metalloenzyme activity & 0.002 \\
\hline & hsa-miR-4756-5p & & negative regulation of trophoblast cell migration & 0.002 \\
\hline & & & $\begin{array}{l}\text { negative regulation of membrane protein ectodomain } \\
\text { proteolysis }\end{array}$ & 0.002 \\
\hline & & & cell activation & 0.005 \\
\hline & & & metalloendopeptidase inhibitor activity & 0.005 \\
\hline & & & platelet alpha granule lumen & 0.005 \\
\hline & & & platelet degranulation & 0.008 \\
\hline & & & extracellular matrix disassembly & 0.012 \\
\hline & & & response to peptide hormone & 0.015 \\
\hline \multirow[t]{5}{*}{ PTX3 } & hsa-miR-29a-3p & KEGG pathway & NA & \\
\hline & hsa-miR-29b-3p & GO terms & NA & \\
\hline & hsa-miR-29c-3p & & & \\
\hline & hsa-miR-101-3p & & & \\
\hline & hsa-miR-144-3p & & & \\
\hline \multirow[t]{10}{*}{ VMP1 } & hsa-miR-19a-3p & KEGG pathway & MicroRNAs in cancer & $3 e-24$ \\
\hline & hsa-miR-19b-3p & & Proteoglycans in cancer & 0.010 \\
\hline & hsa-miR-148a-3p & GO terms & autophagic vacuole membrane & 0.008 \\
\hline & hsa-miR-152-3p & & pre-autophagosomal structure & 0.008 \\
\hline & miR-223-3p & & cell junction assembly & 0.008 \\
\hline & & & regulation of autophagy & 0.008 \\
\hline & & & embryo implantation & 0.009 \\
\hline & & & cell junction organization & 0.014 \\
\hline & & & autophagy & 0.017 \\
\hline & & & single organismal cell-cell adhesion & 0.017 \\
\hline
\end{tabular}

cell line) cells from sepsis-induced AKI model exposed to lipopolysaccharide (LPS) serum compared to control group exposed to phosphate-buffered saline (PBS) serum [37]. The studies suggested $S 100 A 9$ could be potential diagnostic biomarker of sepsis-induced AKI. VMP1, which was first observed in pancreatitis, encoding a multispanning membrane protein in the endoplasmic reticulum, participating in the process of autophagy [38]. SLPI gene encodes a protease inhibitor and regulator of innate and adaptive immunity [39]. It is revealed that the expressed SLPI was elevated in patients with sepsis and the level of elevation was associated with the severity of organ dysfunction [40]. PTX3 is the prototype of the long pentraxin subfamily, playing an important role in regulating humoral innate immune response and participating in innate resistance to pathogens [41]. However, the role of VMP1, SLPI, PTX3 in the development of SAAKI remains unclear. Further studies will be required to identify the relationship between these genes and SSAKI.
miRNA comprise a class of small noncoding RNAs, regulating protein expression through degeneration or inhibition translation when binding to mRNA [42]. Recent studies have shown that miRNAs were closely involved with the occurrence, development, and prognosis of septic AKI [43, 44]. Our study identified several miRNAs targeting each co-DEG involved with sepsis and AKI. Xu et al. found that miR-29b-3p were implicated in PI3K-Akt signaling pathway, suggesting it might be associated with the pathogenesis of sepsis-induced AKI [45]. Ma et al. observed that the expression of miR-152-3p was upregulated in the serum of septic AKI patients and positively related to serum creatinine, urea nitrogen, interleukin $1 \beta$ and tumor necrosis factor $\alpha$, suggesting the extent of miR-152-3p elevation correlated to the severity of kidney injury and inflammatory response [46]. Tan et al. reported that DLX6-AS1 mediated HK-2 cell pyroptosis in LPS-induced AKI via repress miR-223-3p expression in HK-2 cells, showing enhanced miR-223-3p expression 
may serve a new strategy for more effective control of septic AKI [47]. These findings shown that miR-29b-3p, miR-152-3p, and miR-223-3p may be potential targets for the treatment of SSAKI. However, the effect and possible mechanism of other predicted miRNAs in SSAKI is unclear, and further exploration will be required to reveal the correlation between the miRNAs and pathogenesis of SSAKI to provide theoretical support for its clinical treatment.

The limitation of our study is that these identified biomarkers are currently limited to the theoretical level. Further experimental studies and clinical trials should be carried out to obtain accurate verification and validate our results.

\section{Conclusions}

The hub genes of EGF and OLFM4 may be involved in the occurrence and progression of AKI and QPCT, CKAP4, PRKCQ, PLAC8, PRC1, BCL9L, ATP11B, KLHL2, LDLRAP1, NDUFAF1, IFIT2, CSF1R, HGF, NRN1, GZMB, and STAT4 may be associated with septic shock. Besides, co-DEGs of VMP1, SLPI, PTX3, TIMP1, OLFM4, LCN2, and $S 100 A 9$ were identified to link AKI and septic shock. Finally, predicted miRNAs for each co-DEGs may be regarded as potential biomarkers or therapeutic targets for SSAKI, especially miR-29b-3p, miR-152-3p, and miR-223-3p.

\begin{abstract}
Abbreviations
AKI: Acute kidney injury; BP: Biological process; CC: Cellular component; CTD: Comparative toxicogenomics database; DEGs: Differentially expressed genes; eGFR: Estimated glomerular filtration rate; GEO: Gene expression omnibus; GO Gene Ontology; iMCD3: Inner medullary collecting duct cell line; ICU: Intensive care unit; KEGG: Kyoto Encyclopedia of Genes and Genomes; LPS: Lipopolysaccharide; miRNAs: microRNAs; MCODE: Molecular Complex Detection; MF: Molecular function; MMPs: Matrix metalloproteinases; PPI: Protein-protein interaction; PCT: Procalcitonin; PBS: Phosphate-buffered saline; STRING: Search Tool for the Retrieval of Interacting Genes; SSAKI: Septic-shock-associated acute kidney injury; SAAKI: Sepsis-associated acute kidney injury.
\end{abstract}

\section{Supplementary Information}

The online version contains supplementary material available at https://doi. org/10.1186/s41065-021-00176-y.

Additional file 1. Differentially expressed genes involved in AKI samples.

Additional file 2. Differentially expressed genes involved in septic shock samples within 0.5 hours.

Additional file 3. Differentially expressed genes involved in septic shock samples within 24 hours.

Additional file 4. Differentially expressed genes involved in septic shock samples within 48 hours.

Acknowledgements

Not applicable.

\section{Authors' contributions}

$Y T, X Y$ and $H S$ designed the research study. YY and SP took responsibility for statistical analyses. YT and JX wrote the manuscript. YS evaluated and revised the manuscript. All authors read and approved the final manuscript.

\section{Funding}

This work was supported by grants from the National Natural Science Foundation of China (No: 81971818, 81772047, and 82002026).

\section{Availability of data and materials}

All data generated or analysed during this study are included in this published article [and its Supplementary information files].

\section{Declarations}

Ethics approval and consent to participate

Not applicable.

\section{Consent for publication}

Not applicable.

\section{Competing interests}

The authors declare that they have no competing interests.

Received: 7 December 2020 Accepted: 30 March 2021

Published online: 16 April 2021

\section{References}

1. Singer M, Deutschman CS, Seymour CW, Shankar-Hari M, Annane D, Bauer $\mathrm{M}$, et al. The third international consensus definitions for sepsis and septic shock (Sepsis-3). JAMA. 2016;315:801.

2. Huet $O$, Chin-Dusting JP. Septic shock: desperately seeking treatment. Clin Sci. 2014;126:31-9.

3. Plataki M, Kashani K, Cabello-Garza J, Maldonado F, Kashyap R, Kor DJ, et al. Predictors of acute kidney injury in septic shock patients: an observational cohort study. Clin J Am Soc Nephrol. 2011;6:1744-51.

4. Uchino S, Kellum JA, Bellomo R, Doig GS, Morimatsu H, Morgera S, et al. Acute renal failure in critically ill patients: a multinational, multicenter study. JAMA. 2005;294:813-8.

5. Zarjou A, Agarwal A. Sepsis and acute kidney injury. J Am Soc Nephrol. 2011;22:999-1006.

6. Bagshaw SM, George C, Bellomo R. Early acute kidney injury and sepsis: a multicentre evaluation. Crit Care. 2008;12:R47.

7. Bagshaw SM, Uchino S, Bellomo R, Morimatsu H, Morgera S, Schetz M, et al. Septic acute kidney injury in critically ill patients: clinical characteristics and outcomes. Clin J Am Soc Nephrol. 2007;2:431-9.

8. Schrier RW, Wang W. Acute renal failure and sepsis. N Engl J Med. 2004;351:159-69.

9. Neveu $H$, Kleinknecht D, Brivet F, Loirat P, Landais P. Prognostic factors in acute renal failure due to sepsis. Results of a prospective multicentre study. The French study group on acute renal failure. Nephrol Dial Transplant. 1996;11:293-9.

10. Thomas ME, Blaine C, Dawnay A, Devonald MA, Ftouh S, Laing C, et al. The definition of acute kidney injury and its use in practice. Kidney Int. 2015;87:62-73

11. Mishra J, Dent C, Tarabishi R, Mitsnefes MM, Ma Q, Kelly C, et al. Neutrophil gelatinase-associated lipocalin (NGAL) as a biomarker for acute renal injury after cardiac surgery. Lancet. 2005;365:1231-8.

12. Herrera J, Rodríguez-Iturbe B. Stimulation of tubular secretion of creatinine in health and in conditions associated with reduced nephron mass. Evidence for a tubular functional reserve. Nephrol Dial Transplant. 1998;13:623-9.

13. Ichai C, Vinsonneau C, Souweine B, Armando F, Canet E, Clec'H C, et al. Acute kidney injury in the perioperative period and in intensive care units (excluding renal replacement therapies). Ann Intensive Care. 2016;6:48. 
14. Famulski KS, de Freitas DG, Kreepala C, Chang J, Sellares J, Sis B, et al. Molecular phenotypes of acute kidney injury in kidney transplants. J Am Soc Nephrol. 2012;23:948-58.

15. Cazalis MA, Lepape A, Venet F, Frager F, Mougin B, Vallin H, et al. Early and dynamic changes in gene expression in septic shock patients: a genomewide approach. Intensive Care Med Exp. 2014;2:20.

16. Barrett T, Wilhite SE, Ledoux P, Evangelista C, Kim IF, Tomashevsky M, et al. NCBI GEO: archive for functional genomics data sets--update. Nucleic Acids Res. 2013:41:D991-5.

17. Ritchie ME, Phipson B, Wu D, Hu Y, Law CW, Shi W, et al. Limma powers differential expression analyses for RNA-sequencing and microarray studies. Nucleic Acids Res. 2015;43:e47.

18. Shannon P, Markiel A, Ozier O, Baliga NS, Wang JT, Ramage D, et al. Cytoscape: a software environment for integrated models of biomolecular interaction networks. Genome Res. 2003;13:2498-504.

19. Bandettini WP, Kellman P, Mancini C, Booker OJ, Vasu S, Leung SW, et al. MultiContrast delayed enhancement (MCODE) improves detection of subendocardial myocardial infarction by late gadolinium enhancement cardiovascular magnetic resonance: a clinical validation study. J Cardiovasc Magn Reson. 2012;14:83.

20. Ashburner M, Ball CA, Blake JA, Botstein D, Butler H, Cherry JM, et al. Gene ontology: tool for the unification of biology. The gene ontology consortium. Nat Genet. 2000;25:25-9.

21. Altermann E, Klaenhammer TR. PathwayVoyager: pathway mapping using the Kyoto encyclopedia of genes and genomes (KEGG) database. BMC Genomics. 2005;6:60

22. Yu G, Wang LG, Han Y, He QY. clusterProfiler: an R package for comparing biological themes among gene clusters. OMICS. 2012;16:284-7.

23. Davis AP, Grondin CJ, Johnson RJ, Sciaky D, King BL, McMorran R, et al. The comparative toxicogenomics database: update 2017. Nucleic Acids Res. 2017:45:D972-8.

24. Carbon S, Ireland A, Mungall CJ, Shu S, Marshall B, Lewis S. AmiGO: online access to ontology and annotation data. Bioinformatics. 2009;25:288-9.

25. Li JH, Liu S, Zhou H, Qu LH, Yang JH. starBase v2.0: decoding miRNAceRNA, miRNA-ncRNA and protein-RNA interaction networks from largescale CLIP-Seq data. Nucleic Acids Res. 2014;42:D92-7.

26. Paraskevopoulou MD, Georgakilas G, Kostoulas N, Vlachos IS, Vergoulis T, Reczko M, et al. DIANA-microT web server v5.0: service integration into miRNA functional analysis workflows. Nucleic Acids Res. 2013:41:W169-73

27. Batra J, Robinson J, Soares AS, Fields AP, Radisky DC, Radisky ES. Matrix metalloproteinase-10 (MMP-10) interaction with tissue inhibitors of metalloproteinases TIMP-1 and TIMP-2: binding studies and crystal structure. J Biol Chem. 2012;287:15935-46.

28. Bojic S, Kotur-Stevuljevic J, Kalezic N, Stevanovic P, Jelic-Ivanovic Z, Bilanovic D, et al. Diagnostic value of matrix metalloproteinase-9 and tissue inhibitor of matrix metalloproteinase-1 in sepsis-associated acute kidney injury. Tohoku J Exp Med. 2015;237:103-9.

29. Zhang J, Liu WL, Tang DC, Chen L, Wang M, Pack SD, et al. Identification and characterization of a novel member of olfactomedin-related protein family, hGC-1, expressed during myeloid lineage development. Gene. 2002;283:83-93.

30. Li J, Liu C, Li D, Wan M, Zhang H, Zheng X, et al. OLFM4 inhibits epithelialmesenchymal transition and metastatic potential of cervical cancer cells. Oncol Res. 2019;27:763-71.

31. Stark JE, Opoka AM, Mallela J, Devarajan P, Ma Q, Levinsky NC, et al. Juvenile OLFM4-null mice are protected from sepsis. Am J Physiol Ren Physiol. 2020;318:F809-16.

32. Basu RK, Standage SW, Cvijanovich NZ, Allen GL, Thomas NJ, Freishtat $\mathrm{RJ}$, et al. Identification of candidate serum biomarkers for severe septic shock-associated kidney injury via microarray. Crit Care. 2011;15:R273.

33. Goetz DH, Holmes MA, Borregaard N, Bluhm ME, Raymond KN, Strong RK. The neutrophil lipocalin NGAL is a bacteriostatic agent that interferes with siderophore-mediated iron acquisition. Mol Cell. 2002;10:1033-43.

34. Wang M, Zhang Q, Zhao X, Dong G, Li C. Diagnostic and prognostic value of neutrophil gelatinase-associated lipocalin, matrix metalloproteinase-9, and tissue inhibitor of matrix metalloproteinases-1 for sepsis in the emergency department: an observational study. Crit Care. 2014;18:634.

35. Koy M, Hambruch N, Hussen J, Pfarrer C, Seyfert HM, Schuberth HJ. Recombinant bovine S100A8 and A9 enhance IL-1 $\beta$ secretion of interferon-gamma primed monocytes. Vet Immunol Immunopathol. 2013;155:162-70.

36. Chen L, Long $X, X u$ Q, Tan J, Wang G, Cao Y, et al. Elevated serum levels of S100A8/A9 and HMGB1 at hospital admission are correlated with inferior clinical outcomes in COVID-19 patients. Cell Mol Immunol. 2020;17:992-4

37. Leeds J, Scindia Y, Loi V, Wlazlo E, Ghias E, Cechova S, et al. Protective role of DJ-1 in endotoxin-induced acute kidney injury. Am J Physiol Ren Physiol. 2020;319:F654-63.

38. Dusetti NJ, Jiang Y, Vaccaro MI, Tomasini R, Azizi SA, Calvo EL, et al. Cloning and expression of the rat vacuole membrane protein 1 (VMP1), a new gene activated in pancreas with acute pancreatitis, which promotes vacuole formation. Biochem Biophys Res Commun. 2002;290:641-9.

39. Thompson RC, Ohlsson K. Isolation, properties, and complete amino acid sequence of human secretory leukocyte protease inhibitor, a potent inhibitor of leukocyte elastase. Proc Natl Acad Sci U S A. 1986;83:6692-6.

40. Grobmyer SR, Barie PS, Nathan CF, Fuortes M, Lin E, Lowry SF, et al. Secretory leukocyte protease inhibitor, an inhibitor of neutrophil activation, is elevated in serum in human sepsis and experimental endotoxemia. Crit Care Med. 2000;28:1276-82.

41. Bottazzi B, Doni A, Garlanda C, Mantovani A. An integrated view of humoral innate immunity: pentraxins as a paradigm. Annu Rev Immunol. 2010;28:157-83.

42. Sanchez-Mejias A, Tay Y. Competing endogenous RNA networks: tying the essential knots for cancer biology and therapeutics. J Hematol Oncol. 2015;8:30.

43. Shen J, Zhang J, Jiang $X$, Wang H, Pan G. LncRNA HOX transcript antisense RNA accelerated kidney injury induced by urine-derived sepsis through the miR-22/high mobility group box 1 pathway. Life Sci. 2018;210:185-91.

44. Chen Y, Qiu J, Chen B, Lin Y, Chen Y, Xie G, et al. Long non-coding RNA NEAT1 plays an important role in sepsis-induced acute kidney injury by targeting miR-204 and modulating the NF-kB pathway. Int Immunopharmacol. 2018;59:252-60.

45. Xu G, Mo L, Wu C, Shen X, Dong H, Yu L, et al. The miR-15a-5p-XIST-CUL3 regulatory axis is important for sepsis-induced acute kidney injury. Ren Fail. 2019:41:955-66.

46. Ma P, Zhang C, Huo P, Li Y, Yang H. A novel role of the miR-152-3p/ERRFI1/ STAT3 pathway modulates the apoptosis and inflammatory response after acute kidney injury. J Biochem Mol Toxicol. 2020. https://doi.org/10. 1002/jbt.22540.

47. Tan J, Fan J, He J, Zhao L, Tang H. Knockdown of LncRNA DLX6-AS1 inhibits HK-2 cell pyroptosis via regulating miR-223-3p/NLRP3 pathway in lipopolysaccharide-induced acute kidney injury. J Bioenerg Biomembr. 2020;52:367-76.

\section{Publisher's Note}

Springer Nature remains neutral with regard to jurisdictional claims in published maps and institutional affiliations. 